\title{
Atenção Básica à Saúde: aprendendo como passado e refletindo o presente
}

Mateus Aparecido de Faria ${ }^{1}$

\section{Resumo}

A Atenção Básica à Saúde tem como prerrogativa ser o elemento coordenador da rede de atenção à saúde, por conta de sua característica vinculante à comunidade e de estar mais próxima das demandas e necessidades sociais. Nesse sentido, o município de Contagem, Minas Gerais, vem realizando diversas ações com o objetivo de fortalecer esse nível de atenção. Uma dessas ações foi a I Mostra em Atenção Básica à Saúde de Contagem. Assim, o objetivo deste artigo é analisar produções apresentadas nesse evento. A pesquisa foi realizada na perspectiva qualitativa, através do método da educação popular de sistematização de experiências. Os resultados foram apresentados e discutidos seguindo a categorização utilizada na Mostra: Gestão de Unidades e Equipes; Promoção à Saúde e Prevenção de Agravos; Assistência e Tecnologias do Cuidado; Educação Permanente e Formação Profissional; Gestão Participativa e Controle Social e Práticas de Vigilância em Saúde no Território da Atenção Básica. As ações mais frequentes foram aquelas relacionadas à prevenção de agravos, promoção da saúde, assistência em saúde e tecnologias do cuidado. Fatores como apoio da gestão e adesão dos participantes foram facilitadores dos resultados positivos alcançados pelas experiências. Os recursos escassos disponibilizados levaram à restrição da atuação de profissionais de equipes de saúde no fazer-diferente.

\section{Palavras-chave}

Atenção Primária à Saúde. Sistematização. Saúde Pública.

1. Mestrando em Saúde Coletiva pelo Instituto René Rachou, Fundação Oswaldo Cruz, Minas Gerais, Brasil. E-mail: mateusfaria18@gmail.com. 


\title{
Systematization of primary health care experiences in Brazil: learning from the past, reflecting the present
}

Mateus Aparecido de Faria*

\begin{abstract}
Primary Health Care is the coordinator of Health Care network in Brazil because its characteristics make it to be closer to community living and social needs. In this sense, Contagem, Minas Gerais, is a city of Brazilian southern and has been carrying out many actions in order to strengthen Primary Health Care, for example, its First Primary Health Care Exhibition. This article aims to analyze presented papers in this event. It was approached through the qualitative research through systematization of experiences popular education's method. The results were presented and discussed according to the topics used in that event: Management and Working Teams; Health Promotion and Disease Prevention; Health Care and Care Technologies; Continuing Education and Pro-work Training; Popular Management and Social Control and Health Surveillance Practices in Primary Health Care Territory. The most frequent actions were those related to prevention of injuries, health promotion, health care and care technologies. Management support and participants adherence were facilitators factors to positive results of experiences. The lack of available resources led to the restriction of health care workers performance
\end{abstract}

\section{Keywords}

Primary Health Care. Systematization. Public Health.

* MSc student in Public Health, René Rachou Institute, Oswaldo Cruz Foundation, State of Minas Gerais, Brazil. E-mail: mateusfaria18@gmail.com. 


\section{Introdução}

O Sistema Único de Saúde (SUS) é fruto da redemocratização e das lutas do Movimento Sanitário Brasileiro e se encontra em constante construção. Desde o final do século XX, obstina-se a garantir o direito à saúde a toda e qualquer pessoa, indiferente da capacidade de compra ou vínculo empregatício. A Atenção Básica, instalada no Brasil na década de 1990, tem como prerrogativa ser o elemento coordenador da rede de atenção à saúde, por conta de sua característica vinculante à comunidade e, consequentemente, estar mais próxima das demandas e necessidades sociais em saúde (CASTRO; MACHADO, 2012).

O município de Contagem, localizado na Região Metropolitana de Belo Horizonte, Minas Gerais, possui cerca de 650.000 habitantes e o sistema local de saúde abrange mais de 70.000 famílias (SIAB, 2015). Desde 2013, a gestão municipal tem realizado reformas na rede de atenção para garantir o acesso e a qualidade dos serviços à população contagense. Nesse sentido, foi organizado pela Secretaria Municipal de Saúde a I Mostra em Atenção Básica à Saúde de Contagem. O evento contou com a participação de profissionais e usuários dos pontos de saúde e teve como objetivo dar visibilidade às práticas de saúde desenvolvidas nos territórios da Atenção Básica e contribuir para a reflexão e o compartilhamento de ideias e experiências que condissessem com os princípios e diretrizes do SUS. Considerando o volume de informações que circularam na Mostra e a necessidade de agregar tais conhecimentos nas atuais e futuras políticas públicas do município, o objetivo deste artigo é analisar as produções ali apresentadas e relacioná-las com outros trabalhos de âmbito nacional, no sentido de compreender quais avanços o município já conseguiu e quais desafios precisam ainda ser enfrentados.

\section{Metodología}

A pesquisa foi qualitativa de cunho exploratório (MINAYO, 2001). Adotou-se o método popular desistematização de experiências que objetiva produzir conhecimentos a partir da reflexão das próprias práticas (SANTOS; CASTRO, 2014), ou seja, há o reconhecimento de que saber também é do campo da prática e não exclusivo do conceitual. Os processos focalizados foram relacionados às experiências apresentadas na I Mostra em Atenção Básica à Saúde de Contagem, realizada no ano de 2015. Tais experiências se configuram como processos complexos, coletivose sócio-históricos, indoalém da percepção pontual (CASEMIRO et al., 2015), e sua análise pode proporcionar compreendêlas em profundidade e dialogicidade, contribuir para a reflexão teórica entre conhecimentos científicos e aqueles surgidos do trabalho vivo em saúde e influenciar as políticas e o plano de saúde de Contagem-MG.

Foram coletadas todas as 34 experiências expostas na Mostra, sendo 12 apresentadas oralmente e as restantes em formato de pôsteres. A coleta se deu no sítio eletrônico da Prefeitura de Contagem que disponibilizou todos os resumos aprovados para o evento. O registro dos dados se deu primeiramente pela codificação dos resumos das experiências (de R1 a R34). Posteriormente foi construída uma tabela de análise no programa Microsoft Excel 2010 com base em estudos semelhantes ao proposto pela pesquisa (AMORIM, 2010; CASEMIRO et al., 2015), a qual continha colunas denominadas: Título da Experiência, Tema, Objetivos e Avanços/Resultados. O preenchimento da tabela decorreu da identificação dessas informações 
nos resumos, requisitos obrigatórios para apresentação no evento. Por conta da limitação de laudas, não foi possível apresentar a tabela.

A interpretação dos dados foi à luz da literatura recente no campo da saúde, principalmente com aquelas produções que dialogam com os processos de trabalho na Atenção Básica.

\section{Resultados e Discussão}

Os resultados serão apresentados e discutidos seguindo a categorização utilizada na I Mostra: Gestão de Unidades e Equipes; Promoção à Saúde e Prevenção de Agravos; Assistência e Tecnologias do Cuidado; Educação Permanente e Formação Profissional; Gestão Participativa e Controle Social e Práticas de Vigilância em Saúde no Território da Atenção Básica.

\section{Gestão de unidades e equipes}

A gestão em saúde precisa considerar como base do trabalho a trama social na qual está inserida e não apenas tarefas e recursos (ANDRÉ; CIAMPONE, 2007). Considerando a importância dessa questão, a Mostra separou um espaço focando os processos de gestão em Unidades Básicas de Saúde (UBS) e nas Equipes de Saúde da Família.

Após a análise, foram classificados cinco trabalhos. O primeiro (R1) enfoca a resolução de conflitos entre profissionais de uma Unidade Básica de Saúde por meio dos princípios da terapia sistêmica proposta por Bert Hellinger, chamados de "As ordens do amor" (HELLINGER, 2007). Segundo o autor, as relações em sistemas familiar, organizacional ou outros mantêmse em equilíbrio devido a três leis, a saber: 1. Hierarquia, que preconiza o respeito e a atenção às pessoas mais velhas como prioridades, por sustentarem o sistema há tanto tempo; 2. Pertencimento, que reforça a necessidade de qualquer membro do sistema ser reconhecido e respeitado como parte integrante do mesmo; e 3. Equilíbrio de troca, que reconhece que toda pessoa é capaz de oferecer seus recursos e receber o que the for importante para sobrevivência e crescimento dentro do sistema.

Através desse método de psicoterapia grupal, foi possível conhecer as prioridades e necessidades de profissionais da Unidade Básica de Saúde (UBS) e construir junto com a gestão local meios de discutir e resolver tais conflitos. A assunção dos papéis dentro do processo de trabalho em saúde, a valorização dos saberes das pessoas mais antigas na equipe e a gestão compartilhadaforam essenciais para adiminuição dos conflitos nessa UBS. Esse modo de resolver conflitos por meio do diálogo direto entre as partes, seja pela perspectiva sistêmica ou pela educação permanente em saúde, é evidenciado na literatura como uma forma eficaz e ativadora dos encontros entre profissionais e profissionaisusuários (FRANCO; KOIFMAN, 2010). Já a segunda experiência (R5) traz a reorganização da atenção à saúde da mulher em um distrito sanitário de Contagem, a partir da regulação como dispositivo de mudança. Apesar de regulação em saúde ser um termo polissêmico (OLIVEIRA; ELIAS, 2010), há um consenso no sentido de ser um papel de intervenção do Estado, de ordem normativa e administrativa, como relatado nessa experiência. A partir de reuniões e definições coletivas envolvendo gestão e profissionais ginecologistas, o fluxo de atenção foi modificado para uma central de regulação no intuito de verificar os encaminhamentos das UBS para o atendimento especializado e a marcação de consultas. Tais mudanças diminuíram o tempo de espera das mulheres para a consulta ginecológica especializada, priorizando casos mais graves; redução dos encaminhamentos, por conta de 
espaços de formação oferecidos aos profissionais das UBS; eliminação de demanda reprimida para inserção de dispositivo intrauterino; e aumento do vínculo entre profissionais de diferentes esferas de atenção da rede de saúde da mulher.

A reorganização dos processos de trabalho no serviço de vacinas também foi relatada em uma das experiências (R28). A autora e o autor estabeleceram como missão tornar produtivo tal espaço com ações de ampliação do horário de atendimento até a atualização de todos os cartões-espelhos da população cadastrada. Tudo isso só foi possível devido à informatização do setor, à chegada de mais profissionais e à melhoria da infraestrutura da UBS. Tal fato é corroborado por outros estudos (CARVALHO; PEDUZZI; AYRES, 2014; BAIÃO et al., 2014) que destacam que o apoio da gestão municipal do SUS e o investimento em infraestrutura são primordiais para consolidar a prestação do cuidado de qualidade e até mesmo para a resolução de conflitos dentro da equipe de saúde.

O quarto trabalho (R32) relata a experiência da resolução de conflitos entre duas equipes de Saúde da Família (ESF) em uma mesma UBS - uma recém-criada e outra que acompanhava a população há algum tempo. Esse conflito está mais próximo do tipo "Falta de colaboração entre os trabalhadores", uma das seis classificações de Carvalho, Peduzzi e Ayres (2014) e a mais frequente na Atenção Básica. Sua origem está na ruptura da contratualidade normativa, ética, moral e política que é estabelecida entre trabalhadores em seus locais de trabalho. O objetivo da experiência era reorganizar os processos de trabalho devido à implantação dessa nova equipe. Depois de reuniões, foi possível ampliar a oferta de serviços na UBS, além de diminuir a lentidão de processos burocráticos inerentes à administração da Unidade e obter uma articulação eficiente entre as demandas espontânea e programada. Percebe-se que a comunicação em seu sentido forte (CARVALHO; PEDUZZI; AYRES, 2014) foi utilizada para superar os conflitos, isto é, se interrelacionaram de modo a construir acordos válidos para o reestabelecimento da contratualidade no trabalho. Atualmente, busca-se, junto ao Núcleo de Apoio à Saúde da Família (NASF), promover a saúde das equipes de profissionais.

Sob a mesma situação, porém entendida de maneira diferente, está a experiência (R24) de duas ESF em remodelar os processos de trabalho dentro da mesma UBS. Devido à integração entre as ESF, foi possível estabelecer rodízios entre setores e a agenda compartilhada no que foi considerado adequado, ampliando, assim, o acesso aos serviços oferecidos, redução da demanda espontânea e por consulta especializada, produção da autonomia do sujeito e monitoramento e avaliação da atenção prestada. Nesse relato, a coexistência de duas ESF não foi descrita como um conflito em si, mas como uma oportunidade de mudança frente aos novos arranjos organizacionais do SUS.

Percebe-se, pelas experiências inseridas nesse tópico, que o diálogo direto entre diferentes atores da rede de atenção tem potencial para ativar transformações locais no SUS. A identificação do problema, a construção coletiva da solução e a implantação compartilhada das deliberações se constituem em fluxo de ativação do trabalho vivo em saúde, aquele que entende o usuário do sistema como possuidor de direitos que precisam, cotidianamente, ser garantidos e que o ambiente de trabalho também é educativo (MERHY, 2007). O método adotado vai variar de acordo com os saberes das pessoas envolvidas e as situações vivenciadas, porém a essência das experiências é o estabelecimento do diálogo a fim de corresponsabilizar todos os atores envolvidos no processo.

\section{Promoção à saúde e prevenção de agravos}

O binômio promoção/prevenção já é tema de debate nacional há muitos anos (NORMAN; 
TESSER, 2015). Entendido como um complexo conceitual que busca proporcionar qualidade de vida por ações que buscam afastar ou abrandar o surgimento de doenças e outros agravos à saúde, ao mesmo tempo em que eleva o bem-estar do indivíduo em sociedade. Tal binômio ainda é um desafio para o campo da saúde por conta de sua operacionalização com outras tarefas da Atenção Básica como o cuidado clínico e vigilância em saúde. Tal desafio é afirmado, em parte, pelo expressivo contingente de experiências arroladas nessa categoria. Nela foram incluídas 16 experiências, representando quase metade do universo de relatos analisados. Isso corrobora também com a ampliação de ações intersetoriais e de promoção à saúde que vem acontecendo na Atenção Básica nos últimos anos (BRASIL, 2012).

Dentre as temáticas apresentadas, o trabalho com grupos operativos foi o mais presente nessa categoria: nove das dezessete experiências apresentaram seus resultados com os grupos de hipertensos e diabéticos (R8), de gestantes (R14), de pessoas idosas (R3; R13), de tabagismo (R11; R17), de Lian Gong (R19), de asmáticos (R4) ou mesmo na sala de espera da UBS (R30). A ideia de grupo operativo se baseia no pressuposto de que determinadas pessoas passam por experiências e estados parecidos de saúde, colocando-se semelhantes objetivos e, portanto, se reconhecendo e dando suporte umas às outras, tornado o espaço grupal uma estratégia de atendimento das necessidades e anseios, a partir da escuta acolhedora das/ dos participantes (MARON et al., 2014).

As experiências sobre grupo operativo trazem avanços na promoção da saúde e prevenção de agravos de populações específicas: diminuição e até eliminação da demanda reprimida, como aconteceu no grupo destinado às crianças com asma; ampliação da oferta de serviços por conta da diminuição do uso de consultas médicas por parte das pessoas participantes do grupo de idosos; as crises sintomáticas diminuíram nos grupos de asmáticos e de hipertensos; relatos de outros participantes da diminuição do uso de fármacos, inclusive ansiolíticos e antidepressivos por conta das atividades; nos grupos de pessoas idosas, deu-se ênfase à produção da autonomia dos sujeitos, uma vez que a realização das atividades da vida diária pôde ser retomada; em quase sua totalidade foi relatado o fortalecimento do vínculo entre a equipe de saúde e usuários, desencadeando outros movimentos dentro e fora da unidade de saúde como maior adesão da população às propostas da UBS, apropriação de conhecimentos científicos (no caso do grupo de gestantes) e busca pelo aperfeiçoamento por profissionais participantes.

Os resultados alcançados demonstram as vantagens de utilizar o grupo operativo na Atenção Básica, chamados por Lucchese et al. (2013) de saltos qualitativos. Outros estudos (ROTOLI et al., 2012; PEDRONI et al., 2013; MARON et al., 2014) apontam igualmente as contribuições dessa ferramenta de cuidado em saúde e seus benefícios em diferentes contextos sanitários. As limitações do grupo operativo se referem ao seu aspecto local e de difícil influência nas políticas públicas de saúde, mesmo que no município. Porém os relatos já apontam uma saída à característica limitadora quando citam o desejo e os pedidos de replicação dessa estratégia em UBS vizinhas ou do mesmo distrito sanitário. Replicar as experiências bemsucedidas e considerar as especificidades locais pode provocar um movimento de consolidação dessa ação isolada como uma estratégia do município nessa esfera da rede de atenção à saúde, assim como influenciar outros pontos como atenção hospitalar e especializada. O segundo tema apresentado nessa categoria foi em relação à saúde dos/das trabalhadores/as das UBS. Um dos relatos (R20) enfoca a aplicação do Mindfullness Based Stress Reduction (MBSR) em profissionais de enfermagem e agentes comunitários de saúde 
(ACS) por terem passado por um período difícil em relação à comunidade. Essa terapia é conduzida pela total atenção ao momento atual, não utilizando de lembranças ou acontecimentos passados. O intuito é sair do automatismo que, segundo Vandenberghe e Sousa (2006), prejudica a flexibilidade necessária para lidar com momentos difíceis. Tal experiência levou os profissionais que dela participaram a uma redução do estresse e da ansiedade, melhoria das relações familiares e trabalhistas e refletiu positivamente nos exames laboratoriais realizados antes e depois do MBSR.

O outro trabalho (R6) enfocou a saúde das/dos ACS de três equipes de Saúde da Família. Foram realizados encontros com diversas atividades conduzidas por uma psicóloga e uma terapeuta ocupacional, em duas etapas, com intervalo de três meses. Percebeu-se a construção da autovalorização e autoconsciência na qualidade de ACS e profissional de saúde, o que acarretou à melhoria das relações nas equipes de origem. Isso, segundo Costa e Ferreira (2011), é essencial para a efetivação da missão do ACS: ser o elo entre a comunidade e o sistema. $\mathrm{O}$ cuidado com esse profissional faz com que haja melhoria nas condições de atendimento à população, em um movimento de cuidar de quem cuida, fato justificador de diversas experiências com esse importante ator sanitário (GOMES et al., 2010)

A arteterapia na promoção à saúde foi tema de dois trabalhos, um realizado em uma UBS (R12) e outro em um Centro de Convivência (R7). No primeiro, a arteterapia foi utilizada para o cuidado de mulheres consideradas poliqueixosas e/ou com algum sofrimento mental. Vescosi, Souza e Avelar (2015) descrevem-nas como pessoas com sintomas vagos e difusos (SVD) e concluem que elas são representadas como casos cansativos e chatos, que são deixadas de lado por conta da visão biomédica ainda prevalente em grande parte das UBS. As técnicas de produção de artesanato eram socializadas tanto por profissionais de saúde como pelas participantes, proporcionando o compartilhamento e a dialogicidade dos conhecimentos científico e popular. Além disso, aproximou os usuários dos profissionais, desconstruindo a estranheza dos SVD e humanizando o cuidado. Já no segundo, é descrito um caso de um usuário do Centro de Convivência que havia sido encaminhado por uma UBS. Em ambos os casos, a melhoria da autoestima, das relações interpessoais e da qualidade de vida é evidenciada como resultado positivo das experiências, indicando outros rumos para a atenção à saúde mental, consonante ao discurso e às práticas da reforma psiquiátrica brasileira. Ao proporcionar a expressão artística, tais espaços contribuem, como apontado por Damasceno e Reinaldo (2009), para oresgatedoser-cidadãodosusuários.

Estratégias para implantação do Programa Nacional de Controle do Tabagismo (PNCT) em um distrito sanitário foi relatado por outro trabalho (R17), que, por meio do apoio intersetorial e interdisciplinar, demonstrou resultados como ampliação da área de abrangência do Programa, corresponsabilização da gestão local e dos profissionais de saúde quanto à prevenção de agravos e reabilitação dos danos provocados pelo uso do tabagismo e formação de núcleos de tratamento em todo o território distrital. O PNCT surgiu pela iniciativa conjunta entre Instituto Nacional do Câncer, secretarias estaduais e municipais de saúde e setores da sociedade civil, objetivando diminuir o início do hábito de fumar entre jovens e os riscos de tabagismo passivo (LUCCHESE et al., 2013). O arranjo gestão-Estado-sociedade parece ser uma forma eficaz de implantar o PNCT, uma vez que funcionou para a conformação da política em âmbito nacional e alcançou importantes avanços no contexto municipal.

Outra experiência (R25) teve como objetivo apresentar os resultados de uma pesquisa feita com e pelos/as estudantes de uma 
escola municipal contagense sobre a percepção de risco relativo à dengue. Tal iniciativa serviu tanto para levantar dados amostrais sobre os domicílios e o risco de proliferação do mosquito transmissor como também para chamar os/as estudantes à ação, já que foram eles/elas que coletaram os dados de suas próprias casas. Com isso, a apropriação do discurso preventivista e de combate à doença foi construída pelos estudantes-pesquisadores através de um método ativo de aprendizagem, a pesquisa-ação. Outro estudo (ALBUQUERQUE et al., 2014) também reforça o papel de estudante na transformação ambiental necessária, indicando a necessidade de escuta dos adultos para com essa população.

Por fim, a saúde da criança foi tema da experiência (R27) que envolveu toda uma ESF para prestar assistência a crianças de 0 a 6 anos, principalmente aquelas que apresentavam quadro de baixo peso ou sobrepeso. As mudanças dos processos de trabalhos envolveram ações como criação de grupos operativos com gestantes e mães, ampliação da oferta de prescrição de fortalecimento de ferro e dias de atividades lúdicas para a população adscrita. Esse novo cenário de atenção à criança proporcionou fortalecimento de vínculo entre população e equipe, diminuição da presença de crianças na agenda espontânea e alcance de $100 \%$ das crianças dentro da faixa de peso considerada normal. Estudos (CANELLA; NOVAES; LEVY, 2015; MENEZES et al.,2012) apontam que tanto o sobrepeso como o baixo peso de crianças influenciam positivamente nos gastos, tanto familiares quanto governamentais. Sendo assim, estratégias de atuação para o público materno-infantil causam impactos que transcendem o território da UBS e colaboram para a eficiência do SUS.

\section{Assistência e tecnologias do cuidado}

A Atenção Básica, segundo Silva et al. (2015), abarca propostas assistenciais universalistas que possuem como objetivo solucionar problemas de saúde pela oferta de ações preventivistas, curativas, promotoras e reabilitadoras na esfera da saúde e usa de determinadas tecnologias próprias de seu nível de atenção para alcançar as necessidades de sua população.

Nessa categoria, foram agrupadas dez experiências acerca de modos de cuidar da saúde e o uso de tecnologias leves e leveduras. O tema mais frequente apresentado foi relacionado à atenção à Saúde Mental, com cinco trabalhos, a saber: ação coletiva para cuidadores, mães e pais de crianças e adolescentes adscritos a uma UBS (R18); mostra de arte sobre a luta antimanicomial (R21); duas experiências (R22; R23) envolvendo o método da oficina terapêutica e destaque da equidade no campo da Saúde Mental (R31). Apesar de ser comumente atribuída a aparelhos da esfera especializada como os Centros de Atenção Psicossocial, a atenção à saúde mental se encontra melhor amparada pela Atenção Básica, uma vez que o estabelecimento do vínculo com a comunidade e sua cultura é um dos seus princípios de atuação (LEMOS; LEMOS; SOUZA, 2007). Vínculo este que foi relatado como um dos resultados positivos de todas as experiências seja com familiares, no ambiente de trabalho, entre usuário e ESF ou mesmo entre pontos de saúde da rede. Outro resultado exposto foi relativo à diminuição da demanda reprimida para serviços mais especializados de saúde mental. Nesse ponto é importante destacar tal resultado, pois a demanda reprimida, por vezes, tende a refrear a Atenção Básica (SOUSA; RIBEIRO, 2011). Conseguir, a partir de métodos que proporcionem o empoderamento dos participantes e provoquem sua autonomia, que essas demandassejam sanadas na UBS se constitui como avanço considerável para o município.

$\mathrm{O}$ atendimento à pessoa hipertensa e diabética (hiperdia) por meio de grupos de 
educação em saúde foi tema de uma experiência que mostrou que, ao passo que a frequência aumentava, percebiam-se melhorias nos indicadores como pressão arterial, circunferência abdominal e peso dos participantes. Tais resultados podem evitar outros agravos prevalentes na população hipertensa e diabética brasileira como infarto agudo do miocárdio (que afeta $11,6 \%$ do total de pessoas hiperdia), outras cardiopatias (24,2\%), acidente vascular cerebral $(11,6 \%)$ (LIMA et al., (2011). Além disso, a demanda espontânea de casos hipertensos e diabéticos diminuiu consideravelmente.

Alguns trabalhos dessa categoria possuíam interfaces com diferentes temas, comoaconteceu com uma experiência sobre oficina terapêutica (R15) e um grupo de saúde mental (R23), ambas com mulheres. A abordagem perpassou os conhecimentos da saúde mental, da promoção da saúde, da saúde da mulher, entre outros campos. E seus resultados são semelhantes aos expostos anteriormente e também na literatura (CORDEIRO et al., 2012): aumento de autoestima, empoderamento, aumento do vínculo social e, neste caso, produção de renda para as participantes. Esses encontros entre mulheres da comunidade e profissionais de saúde elevam o cuidado em saúde a um nível que, ao mesmo tempo, aumenta o aspecto integral da atenção e oferece o fortalecimento do grupo de mulheres na comunidade, podendo enfrentar as adversidades de forma conjunta, apoiada.

Outra experiência foi a ação coletiva de tratamento da dor lombar (R26). Sabe-se que as causas mais prevalentes de dor crônica que chegam à Atenção Básica são cefaleias e lombalgias, aqui entendidas como quadros dolorosos nas regiões lombares, independente de suas causas (CORDEIRO et al., 2008). No caso da experiência apresentada, por meio de atividades terapêuticas e educativas, foi possível verificar: redução da intensidade da dor em $41,6 \%$, redução da incapacidade em 34,9\% e aumento da qualidade de vida em 38,2\%. Além disso, cerca de $63 \%$ dos participantes relataram ter melhorado as dores nas costas bastante ou totalmente. A assiduidade foi essencial para alcançartaisresultados, umavezqueusuáriosmais frequentes apresentaram melhores resultados.

A última experiência (R29) dessa categoria é a que relata a ação de um grupo multidisciplinar para atuação com adolescentes de diferentes territórios a fim de atender a demanda de pais e mães acerca dos conflitos e dificuldades de relacionamento com seus filhos e filhas. Os encontros foram entre profissionais e adolescentes, variando sobre a presença de responsáveis. Apesar de ser considerada de curta duração, a iniciativa proporcionou o diálogo entre saberes dos atores envolvidos e a ressignificação do papel do adolescer na sociedade. A forma como se deu essa experiência corrobora com as considerações de Oliveira, Egry e Gejer (1997) de que o entendimento da realidade do adolescente por parte de profissionais de saúde deve partir de experiências coletivas, superando a individualização do adolescer.

\section{Educação permanente e formação profissional}

A educação permanente em saúde é uma estratégia pedagógica que considera que o SUS vai além do caráter assistencial: se constitui como um espaço de ensino-aprendizagem, em que os saberes construídos são refletidos a fim de modificar as novas práticas, tudo isso no ambiente de trabalho (CECCIM, 2005). Tal concepção faz parte, juntamente com a educação continuada, com capacitações e outros métodos, da formação profissional, necessária para acompanhar as transformações sociais e necessidades da população brasileira. Dentre as experiências da Mostra, foi classificado um relato de experiência (R9) nessa categoria, referente à criação e implementação de um curso de capacitação em saúde bucal direcionado a ACS. Os resultados relatados 
foram além da apropriação do conhecimento de odontologia em saúde coletiva: houve produção da valorização de seu trabalho pelos próprios Agentes e contou com o envolvimento de toda a ESF, tanto no entendimento da necessidade do conhecimento de saberes odontológicos como também do papel do ACS na equipe. Martins et al. (2015), por meio de outra experiência exitosa com educação permanente em saúde com ACS, indicaram a força de encontros entre esses profissionais de saúde para ecoar por outros espaços de luta cotidiana. Proporcionar tais espaços corrobora com a lógica de multiplicadores de saberes, potencializado pelo trabalho do ACS, o qual já está inserido no território e convive com as necessidades de saúde da população adscrita.

\section{Gestão participativa e controle social}

A origem da temática-foco dessa categoria encontra-se no processo de redemocratização do sistema nacional de saúde e na emergência em garantir que assim permaneça (SOUSA, 2014). Os processos de gestão participativa garantem a mediação entre diversos interesses constantes na sociedade e garantem que as necessidades se conjuguem com as intenções do Estado na biogovernança. Uma das instâncias de negociação na gestão participativa é o controle social, que trabalha por deliberações realizadas por representantes de usuários, majoritariamente, gestores e trabalhadores de saúde.

Nessa categoria foi classificada uma experiência (R10) que relata sobre o Encontro Casa Aberta. Realizado em uma praça da cidade, foi organizado em conjunto com a gestão municipal, sociedade civil e a UBS do local com o objetivo de atuar em rede para atender às demandas sociais em saúde. Segundo as autoras,

o envolvimento da população permite a ela se apropriar de sua saúde e conduz à formação da consciência sanitária, que se estende às demais questões sociais. Nesse processo, alcançam-se amadurecimento político e ampliação da cidadania, essenciais ao desenvolvimento de uma sociedade justa e distante das tradicionais práticas opressivas de poder. (R10, p. 1).

O Casa Aberta se constituiu como uma iniciativa interdisciplinar com vistas à integralidade e que mostra à gestão local do SUS o que precisa ser feito em termos de mudanças epidemiológicas e de intervenção biopsicossocial. Sob a perspectiva do usuário, foi possível compartilhar conhecimentos acerca de seus direitos e deveres, ampliando seu acesso aos serviços prestados na UBS, e proporcionar o convite a ocupar mais os espaços institucionais públicos da saúde.

\section{Práticas de vigilância em saúde no território da Atenção Básica}

O conceito de vigilância em saúde é polissêmico, a depender do tempo e do contexto em que são empregados. De modo geral, compreende três principais noções: junção entre vigilâncias epidemiológica e sanitária; ampliação do objeto, contemplando não apenas as enfermidades de notificação compulsória, mas também análise situacional de saúde de determinados grupos populacionais; e, por fim, a noção de vigilância em saúde, potencial transformadora de determinantes sociais de saúde e doença (FARIA; BERTOLOZZI, 2010).

A saúde do/a trabalhador/a é o enfoque da única experiência (R34) categorizada como referente à vigilância em saúde nos territórios da Atenção Básica. Trata-se de um diagnóstico acerca do perfil produtivo em ambiente domiciliar do município de Contagem e contou com a participação de ACS que acompanham o território amostral. Após aplicação e análise dos dados, verificou-se que o trabalho doméstico e a agricultura familiar são as duas ocupações 
mais frequentes no território. É preciso atentar para dois fatores agravantes nos ambientes de trabalho: a presença de indícios do uso de agrotóxicos pela população, aumentando o risco de intoxicações e câncer; e a presença de atividades que podem levar a lesões por esforço repetitivo e trabalho infantil em algumas atividades. Silva, Araújo e Melo (2013, p. 36) demonstram que "os agricultores, assim como sua família, estão vulneráveis à sua própria prática laboral" devido à falta de um trabalho educativo e formativo para as pessoas que utilizam a agricultura como sustento familiar. Isso inclui o uso indiscriminado de agrotóxicos, o cuidado com a saúde no trabalho rural e até mesmo a qualidade do produto que está sendo vendido.

A vigilância em saúde na Atenção Básica se configura como um rompimento da abordagem reducionista, em que a doença é ligada a um determinado agente e a atenção precisa ser direcionada para essa situação (FARIA; BERTOLOZZI, 2010). A experiência traz a possibilidade de intervenção no ambiente e monitoramento de diversos aspectos conectados com os processos de trabalho e os objetivos da Atenção Básica.

\section{Considerações finais}

A I Mostra em Atenção Básica à Saúde de Contagem contou com a participação de diversos/as profissionais de saúde não apenas dessa esfera de atenção - atores nos níveis especializados participaram desse encontro de ativações para mudanças no contexto sanitário do município. Ativações anteriores ao evento, demonstradas pelos 34 relatos de experiências servem para as ativações futuras, por conta do momento de perceber no outro a possibilidade de transformações do próprio trabalho. Esse movimentodeterideiasemodelos deintervenção destoantes dos já ultrapassados clássicos de atuação unicausal em saúde foi uma das características do conjunto analisado neste artigo.

Outro ponto evidenciado em experiências de diferentes categorias foi a escassez de recursos disponibilizados que levou à restrição da atuação de profissionais de equipes de saúde no fazer-diferente. Mesmo com apoio irrestrito da gestão (seja da UBS ou do município) e o comprometimento de diversos profissionais com seus respectivos saberes, o sucesso de experiências é alcançado quando mais pessoas podem usufruir desse cuidado em saúde de qualidade e significante para as demandas sociais. Para tanto, os recursos materiais e financeiros não podem ser desconsiderados.

As ações mais frequentes foram as relacionadas com prevenção de agravos, promoção da saúde, assistência em saúde e tecnologias do cuidado. Se por um lado esse cenário confirma a relevância e o esforço por parte da Atenção Básica contagense em prestar assistência de qualidade e conseguir saltos qualitativos em diversos aspectos, por outro pode ser um sintoma de desvalorização de outros pontos necessários aos processos de trabalho nessa esfera como o controle social e a educação permanente. Em sentido amplo, compreende-se que esses dois instrumentos podem ser usados para a promoção da saúde e até mesmo integrarem o conjunto de tecnologias de cuidado. No entanto, a existência de categorias específicas tanto para ações do controle social como da educação permanente e existir apenas uma experiência para cada pode sinalizar secundarização dessas categorias no planejamento das ações das UBS. Tal afirmação precisa considerar que, possivelmente, nem todas as experiências exitosas do município foram mostradas no evento, porém existe a possibilidade do não reconhecimento dessas temáticas no cotidiano de trabalho em saúde. Paradoxalmente, os conselhos locais de saúde foram indicados, em alguns relatos, como instituição legitimadora do sucesso das experiências, indicando 
a presença do controle social nas UBS.

O conhecimento construído pela rotina do trabalho vivo em saúde, por vezes, é silenciado pelos saberes da "ciência oficial" ou pela própria rotina das UBS. Eventos como a Mostra vem contra tal situação, atribuindo valor e multiplicando momentos que deram e dão certo em locais de difícil comunicação na cidade.
Este artigo tentou compreender as experiências de forma a propiciar um quadro geral dos avanços e desafios da Atenção Básica e reforça a necessidade de olhares mais em profundidade sobre cada relato, no sentido não perder a riqueza do dia a dia do serviço e tornar mais efetiva as políticas públicas em saúde de Contagem, Minas Gerais.

\section{Referências}

ALBUQUERQUE, O. M. R. et al. Percepção de estudantes de escolas públicas sobre o ambiente e a alimentação disponível na escola: uma abordagem emancipatória. Saúde Soc., São Paulo, v. 23, n. 2, p. 604-615, jun. 2014. doi: http://dx.doi.org/10.1590/S0104-12902014000200020.

AMORIM, E. G. de S. Educação popular em saúde no município de Recife: sistematização de experiências desenvolvidas no DS IV de 2001 a 2010. 2010. 73 f. Monografia (Especialização em Gestão de Sistemas e Serviços de Saúde) - Departamento de Saúde Coletiva, Centro de Pesquisas Aggeu Magalhães, Fundação Oswaldo Cruz. 2010. Disponível em:<http://www.cpqam.fiocruz.br/ bibpdf/2010amorim-egs.pdf>. Acesso em: 14 dez. 2015.

ANDRÉ, A. M.; CIAMPONE, M. H. T. Competências para a gestão de Unidades Básicas de Saúde: percepção do gestor. Rev. Esc. Enferm. USP, São Paulo, v. 41, n. spe, p. 835-840, dez. 2007. doi: http://dx.doi.org/10.1590/S0080-62342007000500017.

BAIÃO, B. S. et al. Acolhimento humanizado em um posto de saúde urbano do Distrito Federal, Brasil. Revista de APS, Juiz de Fora, v. 17, n. 3, p. 291-302, jul./set. 2014. Disponível em: < http:// aps.ufjf.emnuvens.com.br/aps/article/view/1870/820>. Acesso em: 14 dez. 2015.

BRASIL. Política Nacional de Atenção Básica. 2012. Brasília: Ministério da Saúde, 2012, 114 p.

CANELLA, D. S.; NOVAES, H. M. D.; LEVY, R. B. Influência do excesso de peso e da obesidade nos gastos em saúde nos domićlios brasileiros. Cad. Saúde Pública, Rio de Janeiro, v. 31, n. 11, p. 2331-2341, nov. 2015. Disponível em: <http://www.scielosp.org/scielo.php?script=sci arttext\&pid=S0102-311X2015001302331\&lng=en\&nrm=iso > . Acesso em: 28 mar. 2017. doi: http://dx.doi.org/10.1590/0102-311X00184214.

CARVALHO, B. G.; PEDUZZI, M.; AYRES, J. R. de C. M. Concepções e tipologia de conflitos entre trabalhadores e gerentes no contexto da atenção básica no sistema único de saúde (SUS). Cad. Saúde Pública, Rio de Janeiro, v. 30, n. 7, p. 1453-1462, jul. 2014. Disponível em: <http://www. scielosp.org/scielo.php?script $=$ sci_arttext\&pid=S0102-311X2014000801453\&lng =en\&nrm $=\mathrm{i}$ so >. Acesso em: 12 dez. 2015. doi: http://dx.doi.org/10.1590/0102-311X00134613.

CASEMIRO, J. P. et al. Impasses, desafios e as interfaces da educação alimentar e nutricional como processo de participação popular. Trab. Educ. Saúde, Rio de Janeiro, v. 13, n. 2, p. 493514, ago. 2015. Disponível em: <http://www.scielo.br/scielo.php?script=sci_arttext\&pid=S1981- 
77462015000200493\&lng=en\&nrm=iso $>$. Acesso em: 19 Nov. 2015. doi: http://dx.doi. org/10.1590/1981-7746-sip00051.

CASTRO, A. L. B. de; MACHADO, C. V. A política federal de atenção básica à saúde no Brasil nos anos 2000. Physis, Rio de Janeiro, v. 22, n. 2, p. 477-506, jun. 2012. Disponível em:<http:// www.scielo.br/scielo.php?script $=$ sci_arttext\&pid $=$ S0103-73312012000200005\&lng $=p t \& n r m=i$ so>. Acesso em: 13 dez. 2015. doi: http://dx.doi.org/10.1590/S0103-73312012000200005.

CECCIM, R. B. Educação permanente em saúde: descentralização e disseminação de capacidade pedagógica na saúde. Ciência \& Saúde Coletiva, Rio de Janeiro, v. 10, n. 4, p. 975-986, 2005. Disponível em: <http://www.scielo.br/scielo.php?pid=S1413-81232005000400020\&script=sci abstract\&tlng=pt>. Acesso em: 15 dez. 2015. doi: http://dx.doi.org/10.1590/S141381232005000400020.

CORDEIRO, Q. et al. Lombalgia e cefaleia como aspectos importantes da dor crônica na atenção primária à saúde em uma comunidade da região amazônica brasileira. Acta Fisiátrica, Ribeirão Preto, v. 15, n. 2, p. 101-105, 2008. Disponível em: < http://www.actafisiatrica.org.br/detalhe artigo.asp?id=149>. Acesso em: 14 dez. 2015.

CORDEIRO, R. C. et al. Oficinas com mulheres na estratégia saúde da família: promovendo a integralidade do cuidado. Rev. Unincor, Três Corações, v. 10, n. 1, p. 290-296, 2012. Disponível em: < http://revistas.unincor.br/index.php/revistaunincor/article/viewFile/528/pdf>. Acesso em: 14 dez. 2015. doi: http://dx.doi.org/10.5892/ruvrv.2012.101.290296.

DAMASCENO, E. C.; REINALDO, A. M. dos S. Oficinas terapêuticas para hábitos de vida saudável no Centro de Convivência Arthur Bispo do Rosário: relato de experiência. Cogitare Enferm., Curitiba, v. 14, n. 1, jan./mar. 2009. Disponível em <http://www.revenf.bvs.br/scielo. php?script=sci_arttext\&pid $=$ S1414-85362009000100025\&lng $=$ pt\&nrm $=$ iso $>. \quad$ Acesso em: 12 dez. 2015.

FARIA, L. S.; BERTOLOZZI, M. R. A vigilância na atenção básica à saúde: perspectivas para o alcance da Vigilância à Saúde. Rev. Esc. Enferm. USP, São Paulo, v. 44, n. 3, p. 789795, set. 2010. Disponível em: <http:/www.scielo.br/scielo.php?script=sci_arttext\&pid=S008062342010000300034\&lng=en\&nrm=iso $>$. Acesso em: 13 dez. 2015. doi: http://dx.doi. org/10.1590/S0080-62342010000300034.

FRANCO, C. M.; KOIFMAN, L. Produção do cuidado e produção pedagógica no planejamento participativo: uma interlocução com a educação permanente em saúde. Interface (Botucatu), Botucatu, v. 14, n. 34, p. 673-682, set. 2010. Disponível em: <http://www.scielo.br/scielo. php?script $=$ sci_arttext $\&$ pid $=\mathrm{S} 1414-32832010000300016 \& \operatorname{lng}=\mathrm{en} \& \mathrm{nrm}=\mathrm{iso}>. \quad$ Acesso $\mathrm{em}$ : 12 dez. 2015. doi: http://dx.doi.org/10.1590/S1414-32832010005000016.

GOMES, K. de O. et al. O agente comunitário de saúde e a consolidação do sistema único de saúde: reflexões contemporâneas. Physis, Rio de Janeiro, v. 20, n. 4, p. 1143-1164, dez. 2010. Disponível em: $\quad<$ http://www.scielo.br/scielo.php?script=sci_arttext\&pid=S0103-73312010000400005\&ln $\mathrm{g}=\mathrm{en} \& \mathrm{nrm}=\mathrm{iso}>$. Acesso em: 12 dez. 2015. doi: http://dx.doi.org/10.1590/S0103- 
73312010000400005.

HELLINGER, B. Ordens do amor: um guia para o trabalho com constelações familiares. São Paulo: Cultrix, 2007. 418 p.

LEMOS, S. S.; LEMOS, M.; SOUZA, M. da G. G. O preparo do enfermeiro da atenção básica para a saúde mental. Arq. Cien. Saúde, Ribeirão Preto, v. 14, n. 4, p. 198-202, out./dez. 2007. Disponível em: < http://repositorio-racs.famerp.br/racs_ol/vol-14-4/ID227.pdf>. Acesso em: 14 dez. 2015.

LIMA, L. M. de et al . Perfil dos usuários do Hiperdia de três unidades básicas de saúde do sul do Brasil. Rev. Gaúcha Enferm., Porto Alegre, v. 32, n. 2, p. 323-329, jun. 2011. Disponível em: < http:// www.scielo.br/scielo.php?script=sci_arttext\&pid=S1983-14472011000200016\&lng =en\&nrm =i so>. Acesso em: 31 mar. 2017. doi: http://dx.doi.org/10.1590/S1983-14472011000200016.

LUCCHESE, R. et al. A tecnologia de grupo operativo aplicada num programa de controle do tabagismo. Texto \& Contexto: Enferm., Florianópolis, v. 22, n. 4, p. 918-926, out./ dez. 2013. Disponível em: <http://www.scielo.br/scielo.php?script=sci_arttext\&pid=S010407072013000400007\&lng=en\&nrm=iso >. Acesso em: 12 dez. 2015. doi: http://dx.doi. org/10.1590/S0104-07072013000400007.

MARON, L. C. et al. Motivos e repercussões da participação de gestantes em grupo operativo no prénatal. REUFSM, Santa Maria, v. 4, n. 3, p. 519-528, Jul/Set. 2014. Disponível em:<http://cascavel. ufsm.br/revistas/ojs-2.2.2/index.php/reufsm/article/view/10827/pdf > . Acesso em: 14 dez. 2015. doi: http://dx.doi.org/10.5902/2179769210827.

MARTINS, M. L. G. et al. Programa de educação permanente de agentes comunitários de saúde (PEPACS): experiência em Porto Alegre, RS. In: FERLA, Alcindo Antônio et al. (Orgs.). Redes vivas de educação e saúde: relatos e vivências da integração universidade e sistema de saúde. Porto Alegre: Rede Unida, 2015. p. 99-104.

MENEZES, L. O. de et al. O impacto do baixo peso ao nascer relacionado à depressão gestacional para o financiamento federal da saúde pública: uma análise do município de Pelotas, Rio Grande do Sul, Brasil. Cad. Saúde Pública, Rio de Janeiro, v. 28, n. 10, p. 1939-1948, out. 2012. Disponível em: <http://www.scielo.br/scielo.php?script=sci_arttext\&pid=S0102-311X2012001 000012\&lng=pt\&nrm=iso $>$. Acesso em: 28 mar. 2017. doi: http://dx.doi.org/10.1590/S0102311 X2012001000012.

MERHY, E. E. Saúde: a cartografia do trabalho vivo. 3. ed. São Paulo: Hucitec, 2007. 189 p. (Saúde em Debate, 145).

NORMAN, A. H.; TESSER, C. D. Acesso ao cuidado na estratégia saúde da família: equilíbrio entre demanda espontânea e prevenção/promoção da saúde. Saúde Soc., São Paulo, v. 24, n. 1, p. 165179, mar. 2015. Disponível em: < http://www.scielo.br/scielo.php?script=sci_arttext\&pid=S010412902015000100165\&lng=en\&nrm=iso $>$. Acesso em: 13 dez. 2015. doi: http://dx.doi. org/10.1590/S0104-12902015000100013.

OLIVEIRA, M. A. de C.; EGRY, E. Y.; GEJER, D. Adolescer e adoecer: perfil de saúde-doença 
de adolescentes de uma unidade básica de saúde do município de São Paulo. Rev. Latino-Am. Enfermagem, Ribeirão Preto, v. 5, n. 1, p. 15-25, jan. 1997. Disponível em: < http://www.scielo. br/scielo.php?script $=$ sci_arttext\&pid $=$ S0104-11691997000100003\&lng $=p t \& n r m=i s o>. \quad$ Acesso em: 13 dez. 2015. doi: http://dx.doi.org/10.1590/S0104-11691997000100003.

OLIVEIRA, R. R. de; ELIAS, P. E. M. Conceitos de regulação em saúde no Brasil. Rev. Saúde Pública, São Paulo, v. 46, n. 3, p. 571-576, jun. 2012. Disponível em: <http://www.scielo.br/ scielo.php?script $=$ sci_arttext \&pid $=$ S0034-89102012000300020\&lng $=$ en $\& n r m=i s o>. \quad$ Acesso em: 12 dez. 2015. doi: http://dx.doi.org/10.1590/S0034-89102012000300020.

PEDRONI, G. A. M. et al. Assistência de enfermagem prestada à pessoa idosa com hipertensão arterial. Rev. Enferm. Centro-Oeste Min., Divinópolis, v. 3, n. 2, p. 662-669, out. 2013. Disponível em:<http://www.seer.ufsj.edu.br/index.php/recom/article/view/379/429>. Acesso em: 12 dez. 2015.

ROTOLI, A. et al. Grupos operativos: estrategia para el cuidado de enfermeira em un centro de atención psicosocial. Index Enferm, Granada, v. 21, n. 4, oct./dic. 2012. Disponível em: < http:// scielo.isciii.es/scielo.php?script=sci_arttext\&pid=S1132-12962012000300011\&lng =es\&nrm =i so >. Acesso em: 12 dez. 2015. doi: http://dx.doi.org/10.4321/S1132-12962012000300011.

SANTOS, K.; CASTRO, A. M. Sistematizar experiências para pensar a prática na educação social. Rev. Edu. Pop, Uberlândia, v. 13, n. 2, p. 36-48, jul./dez. 2014. Disponível em: <http://www.seer. ufu.br/index.php/reveducpop/article/view/26221/15949>. Acesso em: 31 mar. 2017

SIAB. Sistemas de Informações da Atenção Básica. Informações sobre o município de Contagem. 2015. Disponível em: <http://tabnet.datasus.gov.br/cgi/deftohtm.exe?siab/cnv/SIABFbr.def>. Acesso em: 15 jan. 2016.

SILVA, J. P.; ARAúJO, M. Z.; MELO, L. C. de Q. e. Panorama da vulnerabilidade da saúde do agricultor familiar de São José da Princesa, Paraíba. Rev. Bras. Ciências Saúde, João Pessoa, v. 17, n. 1, p. 29-38, jan. 2013.

SILVA, R. M. M. da etal. A integralidade na assistência à saúde da criança na visão dos cuidadores. Saúde Debate, Rio de Janeiro, v. 39, n. 106, p. 718-729, set. 2015. Disponível em: < http://www.scielo. br/scielo.php?script $=$ sci_arttext\&pid $=$ S0103-11042015000300718\&lng $=$ en\&nrm $=$ iso $>$. Acesso em: 13 dez. 2015. doi: http://dx.doi.org/10.1590/0103-1104201510600030013.

SOUSA, F. R. de. Educação popular em saúde e participação de prostitutas: contribuições para a gestão participativa do SUS. Interface (Botucatu), Botucatu, v. 18, suppl. 2, p. 1568, 2014. Disponível em: $<$ http://www.scielosp.org/scielo.php?script=sci_arttext\&pid=S1414-32832014000701568\&lng= en\&nrm=iso >. Acesso em: 13 dez. 2015. doi: http://dx.doi.org/10.1590/1807-57622013.0406.

SOUSA, A. R. B. de; RIBEIRO, K. S. Q. S. A rede assistencial em fisioterapia no município de João Pessoa: uma análise a partir das demandas da atenção básica. Rev. Bras. Ciências Saúde, João Pessoa, v. 15, n. 3, p. 357-368, 2011. Disponível em: <http://periodicos.ufpb.br/ojs2/index. php/rbcs/article/viewFile/10836/6829> . Acesso em: 14 dez. 2015. Doi: http//: dx.doi.org/10.4034/ RBCS.2011.15.03.11. 
VANDENBERGHE, L.;SOUSA, A.C.A. de. Mindfulnessnasterapias cognitivasecomportamentais. Rev. Bras. Terapias Cognitivas, Rio de Janeiro, v. 2, n. 1, jun. 2006. Disponível em:<http://pepsic. bvsalud.org/scielo.php?script=sci_arttext\&pid=S1808-56872006000100004\&lng =pt\&nrm =i so >. Acesso em: 12 dez. 2015.

VESCOSI, R. G. L.; SOUZA, L. G. S.; AVELLAR, L. Z. Usuários poliqueixosos: representações sociais construídas por médicos da atenção primária à saúde. Psicol. Estud., Maringá, v. 20, n. 1, p. 71-82, jan./mar. 2015. Disponível em: <http://www.periodicos.uem.br/ojs/index.php/PsicolEstud/article/ view/25519/pdf_26>. Acesso em: 31 mar. 2017.

Submetido em 21 de novembro de 2016.

Aprovado em 21 de abril de 2017. 